\title{
Spatial System Identification of a Simply Supported Beam and a Trapezoidal Cantilever Plate
}

\author{
Andrew J. Fleming and S. O. Reza Moheimani, Senior Member, IEEE
}

\begin{abstract}
Dynamic models of structural and acoustic systems are usually obtained by means of either modal analysis or finite element modeling. Detrimentally, both techniques rely on a comprehensive knowledge of the system's physical properties. As a consequence, experimental data and a nonlinear optimization are required to refine the model. For the purpose of control, system identification is often employed to estimate the dynamics from disturbance and command inputs to set of outputs. Such discretization of a spatially distributed system places unknown weightings on the control objective, in many cases, contradicting the original goal of optimal control. This paper introduces a frequency domain system identification technique aimed at obtaining spatially continuous models for a class of distributed parameter systems. The technique is demonstrated by identifying a simply supported beam and a trapezoidal cantilever plate, both with bonded piezoelectric transducers. The plate's dimensions are based on the scaled side elevation of a McDonnell Douglas FA-18 vertical stabilizer.
\end{abstract}

Index Terms-Frequency domain, spatial control, spatial system identification, spatially distributed systems, structural modeling.

\section{INTRODUCTION}

$\mathbf{I}$ N THE ANALYSIS and control of distributed parameter systems, it is of great benefit to possess a spatial model. That is, a model that describes system dynamics over an entire spatial domain. This paper is concerned with the modeling and identification for a class of distributed parameter systems. Such systems include but are not limited to: flexible beams and plates, compound linear structures, slewing structures, and acoustic enclosures.

The motivation for finding such a model lies in both the fields of analysis and synthesis. During analysis, the user may simply wish to observe the mode shapes of the structure, or in a more complete utilization of the model, mathematically estimate the spatial feedback control performance of a system utilizing discrete sensors, actuators, and control objectives. For example, consider [1], where a standard $H_{\infty}$ controller [2], [3] is designed to minimize vibration at a single point on a piezoelectric laminate simply supported beam. A spatial model is required to analyze the overall performance of such a controller. The fact that a point-wise controller is shown to provide good local performance but poor spatial performance leads us to the primary application of spatial models-spatial controller synthesis. A

Manuscript received January 11, 2002. Manuscript received in final form February 4, 2003. Recommended by Associate Editor G. A. Dumont. This work was supported by the Australian Research Council under the Discovery Grant DP-0209396.

The authors are with the School of Electrical Engineering and Computer Science, University of Newcastle, Callaghan NSW 2308, Australia.

Digital Object Identifier 10.1109/TCST.2003.816415 number of standard control synthesis variants have emerged that address the control design of spatially distributed systems with discrete sensors and actuators. Recent examples include: spatial feedforward control [4], spatial resonant control [5], spatial $\mathrm{H}_{2}$ control [6], and spatial $H_{\infty}$ control [1].

The modal analysis procedure has been used extensively throughout the literature for obtaining spatial models of structural [7], [8] and acoustic systems [9]. Its major disadvantage being the requirement for detailed physical information regarding the sensors, actuators, and underlying mechanical system. Practical application typically involves the use of experimental data and a nonlinear optimization to identify unknown parameters such as modal amplitudes, resonance frequencies, and damping ratios. Even in this case, the descriptive partial differential equations must still be solved (as functions of the unknown parameters) to obtain the mode shapes. This may be difficult or impossible for realistic structural or acoustic systems with complicated boundary conditions.

Another popular technique for obtaining spatial models is that of finite-element (FE) analysis [10]. This is an approximate method that results in high-order spatially discrete models. If the dynamics of sensors and actuators are known, the integrated model can be cast in a state space form to facilitate control design and analysis [11]. The approximate nature of FE modeling eliminates the need for solving descriptive partial differential equations. Detailed information regarding the structures material properties and boundary conditions is still required. As with the modal analysis procedure, FE models are usually tuned with experimental data [12].

A considerable literature has also developed on the topic of experimental modal analysis, (see [13] for a compilation of such methods). These methods can be predominantly described as frequency-domain transfer function methods. The system is assumed to consist solely of parallel second-order resonant sections. Sensor, actuator, and additional nonmodal dynamics are neglected. One of the most popular methods, widely used in commercial frequency domain modal analysis packages, is the rational fraction polynomial method [13]. As a transfer function method, the model is poorly conditioned, incorrectly describes the systems zero dynamics [14], and neglects nonmodal dynamics. In addition, all of the mentioned experimental modal analysis techniques neglect the fundamental limitations in spatial sampling, i.e., reconstructed mode shapes can be distorted due to violation of the Nyquist criterion in one and two dimensions.

This paper introduces an efficient and correct method for identifying the above class of systems directly from measured frequency response data. 
We begin by introducing a model structure for one- and two-dimensional systems. The structure is parameterized in terms of the shared dynamics, mode shapes, and feed-through function. In Section III, the spatial sampling limitations for linear and spline reconstruction is discussed. Sections IV and $\mathrm{V}$, deal with the identification of shared system dynamics and spatial functions. Results from the identification of a simply supported beam and trapezoidal cantilever plate are presented in Section VI. Conclusions are drawn in Section VII.

\section{MODELING}

The Lagrangian/modal expansion, or Ritz-Kantorovitch method [7] is commonly used to express the spatial deflection of a distributed parameter system as an infinite summation of modes. The modes are a product of two functions, one of the spatial coordinate vector $\mathbf{r}$, and another of the temporal $t$

$$
d(\mathbf{r}, t)=\sum_{i=1}^{\infty} q_{i}(t) \phi_{i}(\mathbf{r})
$$

where the $q_{i}(t)^{\prime} \mathrm{s}$ are the modal displacements, the $\phi_{i}(\mathbf{r})^{\prime} \mathrm{s}$ are the system eigenfunctions, $d(\mathbf{r}, t)$ is the displacement at a point, and $\mathbf{r} \in \mathcal{R}$ is a coordinate vector on the spatial domain $\mathcal{R}$. The mode shapes $\phi_{i}(\mathbf{r})$ must form a complete coordinate basis for the system, satisfy the geometric boundary conditions, and for analytic analysis be differentiable over the spatial domain to at least the degree required by the describing partial differential equations. Many practical systems also obey certain orthogonality conditions.

As discussed in [7], the model (1) can also be expressed in the frequency domain

$$
G_{y}(\mathbf{r}, s)=\sum_{i=1}^{\infty} \frac{F_{i} \phi_{i}(\mathbf{r})}{s^{2}+2 \zeta_{i} \omega_{i} s+\omega_{i}^{2}}
$$

where $G_{y}(\mathbf{r}, s)$ is the transfer function from an external force, or for the system considered in this paper, the applied piezoelectric voltage to the displacement at a point $\mathbf{r}$,

For practical reasons, (2) is often truncated to include only a certain number of modes that approximate the response over a limited bandwidth. [14] introduces a model reduction technique for systems that satisfy certain modal orthogonality conditions. The following truncated model structure is proposed:

$$
\widetilde{G}_{y}(\mathbf{r}, s)=\sum_{i=1}^{N} \frac{F_{i} \phi_{i}(\mathbf{r})}{s^{2}+2 \zeta_{i} \omega_{i} s+\omega_{i}^{2}}+\sum_{i=N+1}^{\infty} k_{i} \phi_{i}(\mathbf{r})
$$

where (referring to [14]) the $k_{i}$ terms are found by minimizing the spatial $\mathcal{H}_{2}$ norm of the resulting error system $\left(\omega_{c}\right.$ is the retained bandwidth)

$$
k_{i}=\frac{F_{i}}{2 \omega_{c} \omega_{i}} \ln \left(\frac{\omega_{i}+\omega_{c}}{\omega_{i}-\omega_{c}}\right) .
$$

We define the model of a general single input spatially distributed system as

$$
\widehat{G}_{y}(\mathbf{r}, s)=H(s)\left[\sum_{i=1}^{N} \frac{\Phi_{i}(\mathbf{r})}{s^{2}+2 \zeta_{i} \omega_{i} s+\omega_{i}^{2}}+D(\mathbf{r})\right]
$$

where $H(s)$ is the concatenation of all nondistributed transfer functions, $\Phi_{i}(\mathbf{r})$ is the $i$ th mode shape, and $D(\mathbf{r})$ is the feedthrough function included to compensate for all higher order truncated contributions to zero dynamics. The filter $H(s)$ is used to model the additional dynamics of sensors, actuators, and for example, antialiasing filters. In this work $H(s)$ is not identified automatically.

The objective will be to identify the parameters $\theta=\left[\begin{array}{llll}\Phi_{i}(\mathbf{r}) & D(\mathbf{r}) & \zeta_{i} & \omega_{i}\end{array}\right]$ from a number of measured spatially distributed point-wise frequency responses

$$
\begin{aligned}
& G_{y}(\mathbf{r}, j \omega) \mathbf{r} \in\left\{\mathbf{r}_{1}, \ldots, \mathbf{r}_{N_{r}}\right\} \in \mathcal{R} \\
& \omega \in\left\{\omega_{1}, \ldots, \omega_{N_{\omega}}\right\}
\end{aligned}
$$

where $N_{r}$ is the number of measured spatial locations and $N_{\omega}$ is the number of measured frequency points per location.

The system (5) has a corresponding state-space representation

$$
\begin{aligned}
\dot{\mathbf{x}}(t) & =\mathbf{A x}(t)+\mathbf{B} u(t) \\
d(\mathbf{r}, t) & =\mathbf{C}(\mathbf{r}) \mathbf{x}(t)+D(\mathbf{r}) u(t)
\end{aligned}
$$

where $\mathbf{C}(\mathbf{r})=\left[\begin{array}{lllll}\Phi_{i}(\mathbf{r}) & 0 & \ldots & \Phi_{N}(\mathbf{r}) & 0\end{array}\right], \quad \mathbf{B}=$ $\left[\begin{array}{lllll}0 & 1 & \cdots & 0 & 1\end{array}\right]^{\mathbf{T}}, D(\mathbf{r})$ is a scalar function of $\mathbf{r}, N$ is the number of modes to be identified, and

$$
\begin{aligned}
\mathbf{A} & =\left[\begin{array}{ccccc}
0 & 1 & & 0 & 0 \\
-\omega_{1}^{2} & -2 \zeta_{1} \omega_{1} & & 0 & 0 \\
& & \ddots & & \\
0 & 0 & & 0 & 1 \\
0 & 0 & & -\omega_{N}^{2} & -2 \zeta_{N} \omega_{N}
\end{array}\right] \\
& \in \mathbf{R}^{2 N \times 2 N} .
\end{aligned}
$$

\section{SPATIAL SAMPLING}

Considering the model structure (5), the spatial functions $\Phi_{i}(\mathbf{r})$ and $D(\mathbf{r})$ must be reconstructed from their identified samples. For a uniformly sampled one-dimensional system, the samples of our continuous functions $\Phi_{i}(\mathbf{r})$ and $D(\mathbf{r})$ are

$$
\begin{array}{ll}
\Phi_{i}(r) & r=n \Delta r \in \mathcal{R} \\
D(r) & n \in\left\{0,1, \ldots, N_{r}\right\}
\end{array}
$$

where the scalar $r$ specifically denotes a one-dimensional system, and $\Delta r$ is the spatial sampling interval.

There are a number of options available for reconstructing the continuous functions, two of which are, traditional linear reconstruction, and spline reconstruction. The following Sections III-A and B, examine the application of each technique to the two cases of band-limited and nonband-limited functions. The aim is to quantify the expected mean square difference between the original continuous function and its corresponding reconstruction. This will allow us to evaluate the required spatial sampling interval as a function of the permissible error. An example of this procedure is performed for a simply supported beam in Section III-C. 


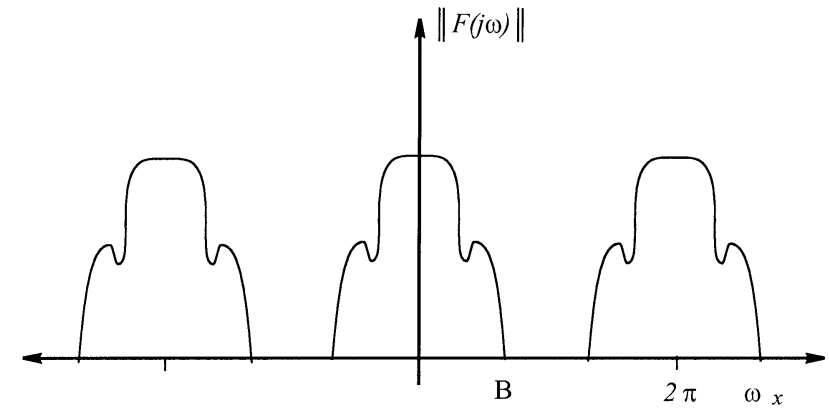

Fig. 1. Discrete magnitude spectra of an oversampled band-limited function.

\section{A. Whittaker-Shannon Reconstruction}

The discrete magnitude spectra of a band limited spatial function $f(r)$ is shown in Fig. 1. To satisfy the Nyquist sampling criterion, the spatial sampling frequency $2 \pi / \Delta r$ (in $\mathrm{rad} / \mathrm{m}$ ) must be greater than twice the highest frequency component of $f(r)$ [15]. Shannon's reconstruction theorem states that $f(r)$ can be reconstructed from its samples

$$
f(r)=\Delta r \sum_{k=-\infty}^{\infty} f(k \Delta r) \frac{\sin \left(\frac{\pi}{\Delta r}(r-k \Delta r)\right)}{\pi(r-k \Delta r)} .
$$

Theoretically, a perfect reconstruction is possible, however in practice, there are two significant sources of degradation.

- For finite time signals, truncating the summation (10) introduces a systematic truncation error. Expressions for bounding the truncation error and references to relevant work can be found in [15].

- In many practical situations, the samples will also contain an additive stochastic disturbance. An expression for the mean integral squared reconstruction error (MISE) experienced when recovering a signal from its corrupted samples can be found in [16]. It is also shown that Shannon reconstruction is not a consistent estimator for band-limited signals recovered from noisy samples, i.e., as the number of signal samples approaches $\infty$, the MISE does not approach zero, in fact the error diverges and also approaches $\infty$. Convergent estimators for such scenarios can be found in [17] and [18].

In general, the spatial function $f(r)$ will not be band-limited. Examples include, the mode shapes of a cantilever beam [8], and the feed-through function for a simply supported beam [14]. Since the samples are obtained indirectly from point-wise frequency response data, no form of low pass filtering is possible. The objective of the following will be to quantify the under-sampling error as a function of the spatial sampling interval.

In their paper reviewing sources of error in linear reconstruction, Thomas and Liu [19] present an expression for the mean square reconstruction error as a function of the power spectral density outside the Nyquist range. The following expression assumes the absence of the optimal low-pass filter, which in our application, cannot be applied to the continuous signal

$$
\left\|f(r)-Q^{s} f(r)\right\|_{2}=\left[\frac{1}{\pi} \int_{\left|\omega_{r}\right|>\pi / \Delta r}\left|F\left(j \omega_{r}\right)\right|^{2} d \omega_{r}\right]^{1 / 2}
$$

where $Q^{s} f(r)$ is the Shannon representation of the sampled function, $\omega_{r}$ is the spatial frequency in radians per meter, and $\left|F\left(j \omega_{r}\right)\right|^{2}$ is the power spectral density of $f(r)$. In the case where the optimal prefilter can be applied, the right-hand side of (11) is reduced by one half.

\section{B. Spline Reconstruction}

In recent years, splines have been recognized for their usefulness in curve and surface fitting problems [20], [21]. A function $f(r)$ can be approximately reconstructed from a spline basis $\varphi(r)$, with coefficients $c(k)$ derived from $f(k \Delta r)$.

$$
\stackrel{s p}{Q}^{n} f(r)=\sum_{k \in Z} c(k) \varphi^{n}\left(\frac{r}{\Delta r}-k\right)
$$

where $c(k) \in l_{2}$ are the (finite square summable) spline coefficients, $Q^{n} f(r)$ is the spline reconstruction of $f(r)$, and $\varphi^{n}(r)$ is the spline generating function. We will limit our choice of generating functions to the $n$th degree $\beta$-splines (of order $n+1$ ) [21]. The condition $c(k) \in l_{2}$ ensures that $Q^{n} f(r)$ is a well-defined subspace of $L_{2}$, the set of square integratable functions, a considerably larger space than the traditional Shannon space of band limited functions. References [22] and [23] present a unified sampling theory for a wide class of approximation operators. In likeness to the Shannon sampling theorem, the optimal spline reconstruction involves an optimal prefiltering of the continuous signal before sampling and reconstruction by the chosen spline basis. The results in this area, including expressions for the rms error, are summarized in [21]. The technique of quantitative Fourier analysis can be applied to quantify the rms reconstruction error [24]. The sampling phase averaged error is given by

$$
\begin{aligned}
& \left\|f(r)-\stackrel{s p}{Q^{n}} f(r)\right\|_{2} \\
& \quad=\left[\frac{1}{2 \pi} \int_{-\infty}^{\infty}\left|F\left(j \omega_{r}\right)\right|^{2} E^{n}\left(\Delta r \omega_{r}\right) d \omega_{r}\right]^{1 / 2}
\end{aligned}
$$

where $E^{n}\left(\Delta r \omega_{r}\right)$ is defined as the frequency error kernel and is a function of the interpolant and $\Delta r$. Analytic expressions for $E^{n}\left(\Delta r \omega_{r}\right)$ have been given for the $\beta$-splines of order up to 6 [24].

In our application, where there is no access to the continuous signal, we cannot apply the optimal prefilter nor achieve the optimal (least squares) fit by projecting our signal onto the approximation space [21]. Instead, we shall simply perform an interpolation. The penalty in doing so is illustrated in Fig. 2, where the error kernels $E^{n}\left(\omega_{r}\right)$ for spline and Shannon reconstruction, optimal and interpolation, are shown for $\Delta r=1$. It can be observed that although the spline interpolant error is globally greater than that of the projector, within the Nyquist range $\left|\omega_{r}\right|<\pi$ the difference is slight. In analogy to Shannon reconstruction, for frequencies beyond the Nyquist rate, the magnitude of the spline interpolant error kernel approaches twice that of the projector.

The spline basis functions also have some interesting variational properties. It is well known that interpolation by the 


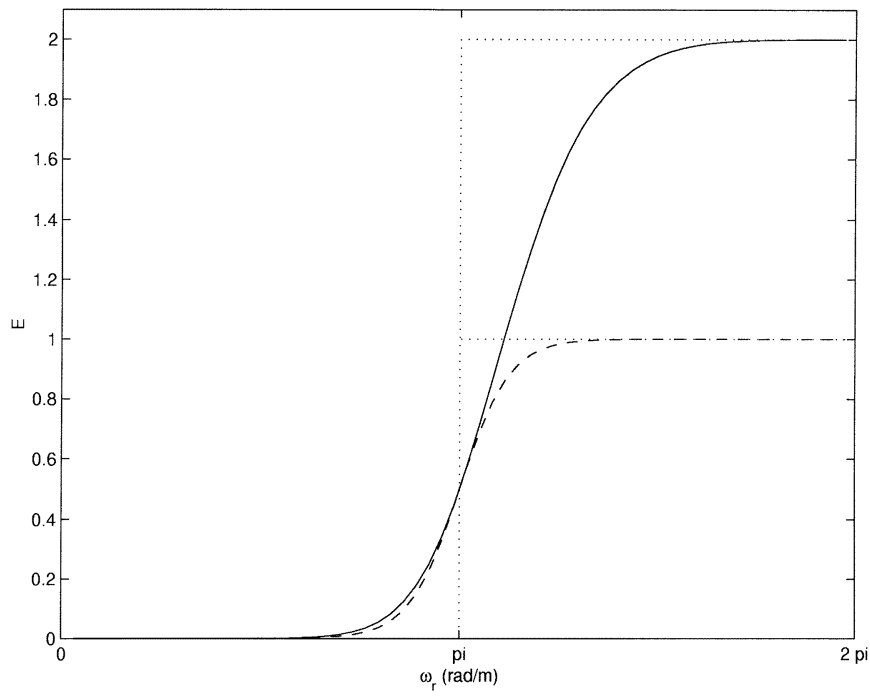

Fig. 2. Unit sampled, cubic spline error kernals. Optimal (with prefilter) (- -), interpolation (-). Shannon reconstruction ...E $E(|\omega|>\pi)=1$, interpolation (...) $E(|\omega|>\pi)=2$.

Shannon basis in the presence of effects such as truncation or additive high-frequency sample noise, tend to result in an overly "peaky" or oscillatory reconstruction. In contrast, spline interpolation (in a certain sense [21], [25]) is the interpolant that oscillates the least. The cubic spline is a special case, as it minimizes the 2-norm of error second derivative, it possesses the property of minimum curvature [21]. As this property is also shared by constrained thin elastic beams and plates, it is natural to reason that cubic splines may be well suited to approximating mechanical functions such as the mode shapes of a simply supported beam.

In the case of noisy samples, we can achieve some degree of immunity by relaxing the interpolation condition and imposing a smoothness constraint. For example, for the cubic splines, by minimizing

$$
\sum_{k}\left(f(k \Delta r)-\stackrel{s p}{n}^{n} f(k \Delta r)\right)^{2}+\lambda \int_{0}^{L}\left(\frac{d^{2} Q^{n} f(r)}{d r^{2}}\right)^{2}
$$

where the second term is a measure of the smoothness. The parameter $\lambda$ is based on the additive noise variance [21].

\section{Spatial Sampling of a Simply Supported Beam}

This section demonstrates how the results presented in Section III-B can be applied to spatial systems. We present an example analysis for a simply supported beam. The objective is to arrive at a point where (11) and (13) can be applied. Both expressions require only the function's power spectral density.

1) Mode Shapes: The mode shapes of a simply supported beam are given by [7]

$$
\phi_{i}(r)=\sqrt{\frac{2}{\rho A_{r} L}} \sin \left(\frac{i \pi r}{L}\right)=\alpha \sin \left(\frac{i \pi r}{L}\right)
$$

where $\rho$ is the material density, $A_{r}$ is the cross-sectional area, and $L$ is the length of the beam. The spatial spectra of $\sum_{i=1}^{N} \phi_{i}(r)$ is impulsive and easily determined

$$
\begin{aligned}
\mathcal{F} & \left\{\sum_{i=1}^{N} \phi_{i}(r)\right\} \\
& =j \pi \sqrt{\frac{2}{\rho A_{r} L}} \sum_{i=1}^{N}\left[\delta\left(\omega_{r}+\frac{i \pi}{L}\right)-\delta\left(\omega_{r}-\frac{i \pi}{L}\right)\right] .
\end{aligned}
$$

The highest frequency component of $\phi_{i}(r) i \in\{1, \ldots, N\}$ is $N \pi / L$, thus, if we were to apply Shannon's theorem ${ }^{1}$ to reconstruct $N$ mode shapes of a simply supported beam

$$
\frac{2 \pi}{\Delta r}>2 \frac{N \pi}{L}, \text { that is, } \Delta r<\frac{L}{N} .
$$

This simple and complete result applies in general to a subclass of the systems (2). Such systems are characterized by sinusoidal mode shapes. Examples include uniform beams and strings in one dimension, plates in two dimensions, and closed acoustic systems in three dimensions.

2) Feed-Through Function $D(r)$ : The feed-through function $D(r)$ can be found analytically for systems of the form (2).

$$
D(r)=\sum_{i=N+1}^{\infty} k_{i} \phi_{i}(r)
$$

where $\phi_{i}(r)$ is given by (15), and $k_{i}$ is given by (4). We can think of (18) as being equivalent to the Fourier series

$$
D(r)=\sum_{i=-\infty}^{\infty} c_{i} e^{(j 2 \pi i r) / T_{r}}
$$

where $T_{r}=2 L$ is the period of repetition

$c_{i}= \begin{cases}\frac{j}{2} \alpha \frac{F_{i}}{2 \omega_{c} \omega_{i}} \ln \left(\frac{\omega_{i}+\omega_{c}}{\omega_{i}-\omega_{c}}\right), & i \in\{\ldots,-N-2,-N-1\} \\ 0, & i \in\{-N, \ldots, N\} \\ \frac{-j}{2} \alpha \frac{F_{i}}{2 \omega_{c} \omega_{i}} \ln \left(\frac{\omega_{i}+\omega_{c}}{\omega_{i}-\omega_{c}}\right), & i \in\{N+1, N+2, \ldots\} .\end{cases}$

The complex coefficients $c_{i}$ reveal the spatial Fourier transform of $D(r)$

$$
\begin{aligned}
\mathcal{F}\{D(r)\} & =\mathcal{F}\left\{\sum_{i=N+1}^{\infty} k_{i} \phi_{i}(r)\right\} \\
& =\sum_{i=-\infty}^{\infty} 2 \pi c_{i} \delta\left(\omega_{r}-i \omega_{f}\right) \\
\omega_{f} & =\frac{2 \pi}{T_{r}}=\frac{\pi}{L} \\
\mathcal{F}\{D(r)\} & =\sum_{i=-\infty}^{\infty} 2 \pi c_{i} \delta\left(\omega_{r}-i \frac{\pi}{L}\right) .
\end{aligned}
$$

Immediately, by the properties of the Fourier transform, we learn some characteristics of the feed-through function $D(r)$.

- As verification, $\mathcal{F}\{D(r)\}=d\left(j \omega_{r}\right)=d\left(-j \omega_{r}\right)^{*} \Leftrightarrow$ $\operatorname{Im}\{D(r)\}=0$ which is known a priori. $\operatorname{Re}\left\{d\left(j \omega_{r}\right)\right\}=$

${ }^{1}$ Neglecting truncation errors. 
0 and $\left|d\left(j \omega_{r}\right)\right|$ are even functions of $\omega_{r}$ [26], $\operatorname{Im}\left\{d\left(j \omega_{r}\right)\right\}$ is an odd function of $\omega_{r}$ [26].

- Since $\mathcal{F}\{D(r)\}$ is purely imaginary, $D(r)$ is an odd function. It is true in general that $\operatorname{Re}\left\{d\left(j \omega_{r}\right)\right\}=0 \Leftrightarrow D(r)=$ $-D(-r)[26]$.

- $D(r)$ is periodic with period $2 L$.

As $\mathcal{F}\{D(r)\}$ does not have compact support on the interval $(-j \infty, j \infty), D(r)$ can not be exactly reconstructed with any finite number of samples. It is also obvious from (24) that the spectra of $D(r)$ lies completely outside the bandwidth of the mode shapes, thus dictating the spatial sampling requirements of the system.

We can now apply (13) to determine the required spatial sampling interval. For a periodic signal $g(r)$, the energy density per unit frequency is given by [27]

$$
|G(f)|^{2}=T \sum_{n \in Z}\left|c_{n}\right|^{2} \delta\left(f-n \frac{1}{T}\right)
$$

where $T$ is the period, $G(f)$ denotes the Fourier transform, and $c_{n}$ are the Fourier coefficients of $g(r)$. By making a change of variables, we can find the power spectral density of $D(r)$

$$
|\mathcal{F}\{D(r)\}|^{2}=2 \pi T_{r} \sum_{i \in Z}\left|c_{i}\right|^{2} \delta\left(\omega_{r}-i \frac{2 \pi}{T_{r}}\right) .
$$

Hence, from (13), the error in reconstructing $D(r)$ from an $n$ th-order spline basis

$$
\begin{aligned}
\left\|D(r)-\stackrel{s p}{Q}^{n} D(r)\right\|_{2}= & {\left[2 L \int_{-\infty}^{\infty}\left(\sum_{i \in Z}\left|c_{i}\right|^{2} \delta\left(\omega_{r}-i \frac{\pi}{L}\right)\right)\right.} \\
& \left.\times E^{n}\left(\Delta r \omega_{r}\right) d \omega_{r}\right]^{1 / 2} \\
= & {\left[2 L \sum_{i \in Z}\left|c_{i}\right|^{2} E^{n}\left(i \pi \frac{\Delta r}{L}\right)\right]^{1 / 2} }
\end{aligned}
$$

where $\stackrel{s p}{Q}^{n} D(r)$ is the spline reconstruction of $D(r)$. The error kernel for a cubic spline $E^{3}(i \pi \Delta r / L)$ is plotted together with the equivalent Shannon kernel in Fig. 2.

We can also apply Parseval's equality to find the mean square value of $D(r)$ over one period

$$
\sum_{i=-\infty}^{\infty}\left|c_{i}\right|^{2}=\frac{1}{2 L} \int_{-L}^{L}|D(r)|^{2} d x
$$

We now consider a specific example: the simply supported beam described in Section VI-A, where three modes are retained for identification. The feed-through function resulting from an analytic model [28] is shown in Fig. 3. The rms value of the reconstruction error $\left(L_{2}\right.$ norm on $\left.[-L, L]\right)$ is plotted against the sampling interval $\Delta r$ in Fig. 4. As the sampling interval increases, the rms error approaches the rms value of the continuous function. $^{2}$ This plot can be used to select a spatial sampling interval that achieves some error specification on $D(r)$.

\footnotetext{
${ }^{2}$ In this analysis we have considered $D(r) \notin L_{2}$. This arises from the periodic nature of the mode shapes. When we refer to the rms or mean square value of such signals, we are implicitly referring to the rms or mean-square value over a single period.
}

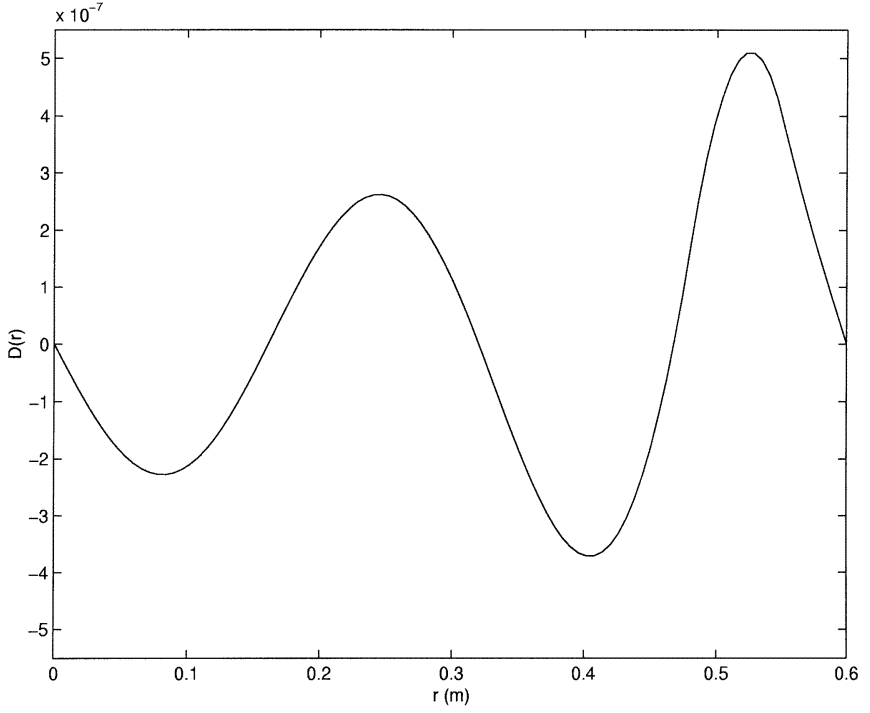

Fig. 3. Analytic feed-through function for the beam described in Section VI-A.

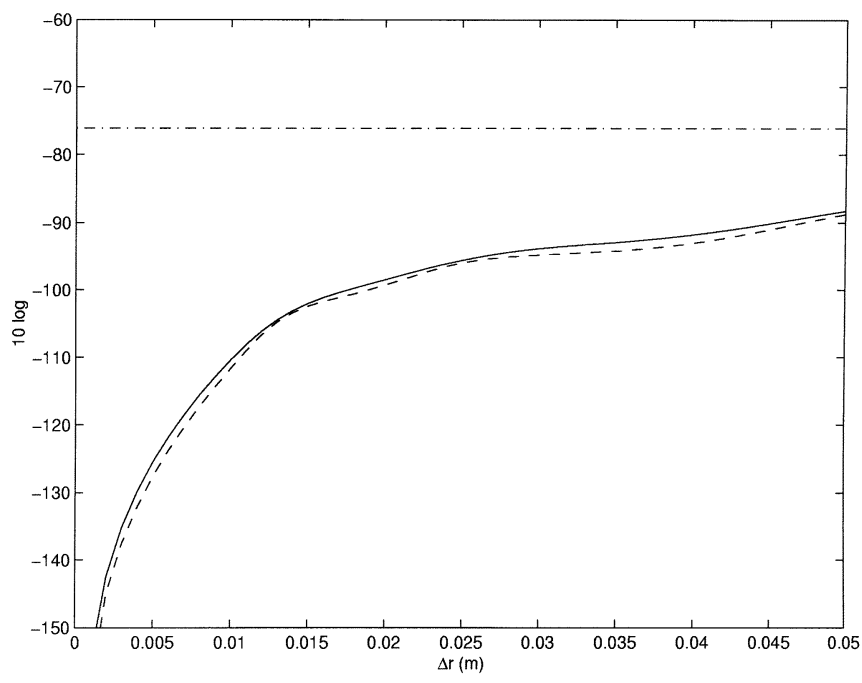

Fig. 4. $\left\|D(r)-Q^{n} D(r)\right\|_{2}$ rms reconstruction error plotted against the spatial sampling interval $\Delta r$. The dashed-dot line indicates the rms value of the function $D(r)$.

3) Other Considerations: The above analysis has considered only a one dimensional system. The Shannon sampling theorem is easily extended to multi-variate functions [15]. By using tensor-product basis functions, spline sampling theory is extended in a similar fashion [21]. Both techniques require an equidistance sampling grid and are based on the application of univariate results in each dimension. For irregular sampling and other complicated reconstructions (e.g., by blending functions [20], or FE methods [20]) no such results are known.

In Section III-C, the sampling limitations for a simply supported beam have been derived. Even when the mode shapes are known a priori, this analysis can be difficult to perform. For the practitioner, we offer a rough rule of thumb.

1) Estimate, by means of a similar system or FE analysis, the highest significant spatial frequency component of the highest order mode to be identified.

2) Consider the feed-through function $D(\mathbf{r})$. Assume that its highest significant frequency component is three times 
that estimated in Step 1). (This step is suggested on the experience of studying and identifying a number of such systems.)

3) Sample the structure as would be done in practice for a function with spatial bandwidth derived in Step 2). Taking into consideration the limited domain of the structure, (allowing for truncation errors), this would normally be from two to five times the rate suggested by the Nyquist criterion.

\section{IDENTIFYING THE SYSTEM MATRIX}

The first step in the identification procedure is to obtain an estimate for $\mathbf{A}$, the system matrix whose eigenvalues reveal the parallel dynamics of each mode. On first inspection, this problem may appear trivial as the transfer function obtained from a single frequency response would perform the task.

For spatially distributed systems we must redefine our measures of model quality and stochastic performance. In essence, the two main sources of error in the identification arise from measurement noise and slight changes in system dynamics over the spatial domain. Intuitively, we would like to distribute the resulting model error in a similar, equally distributed fashion. The task of quantifying such errors is the subject of current research.

The problem can be cast as a multi-input-multi-output (MIMO) system identification problem where each point is regarded as a single output. In the case of a two-dimensional system, where a large number of point-wise frequency response measurements are available, it may be necessary to limit the data space by selecting only a subset of the available points. The virtual system as seen by the system identification algorithm has a single input and $\widetilde{N}_{r}$ outputs, where $\widetilde{N}_{r}$ may be equal to $N_{r}$ or less than $N_{r}$ if the data set is to be truncated. The frequency response of such a system is similar to (6) and can be expressed as

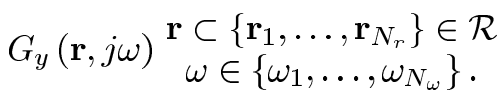

For generality, we treat the identification algorithm as a general matrix function of the data, i.e., $\mathbf{A}=\mathbf{f}\left(\mathbf{G}_{y}(\mathbf{r}, \mathbf{j} \omega)\right)$.

Methods that identify state-space models by exploiting geometric properties of the input and output sequences are commonly known as subspace methods. These methods have received considerable attention in the literature, (see [29] for a survey of time-domain methods). The reader is referred to [30] and [31] for a full discussion of frequency domain techniques. Frequency domain subspace-based algorithms have proven particularly useful for identifying high-order multi-variable resonant systems [32].

\section{IDENTIFying THE Mode Shapes AND FEED-THROUGH FUNCTION}

Samples of the spatial modal and feed-through functions are first identified from the frequency response data and system matrix. The continuous functions are then approximated by linear or spline reconstruction.

\section{A. Identifying the Samples}

Samples of the spatial functions will now be identified from the available frequency response data.

Definitions: The spatial response matrix

$$
\mathbf{G}=\left[\begin{array}{ccc}
G_{y}\left(\mathbf{r}_{1}, j \omega_{1}\right) & \cdots & G_{y}\left(\mathbf{r}_{N_{r}}, j \omega_{1}\right) \\
\vdots & \ddots & \vdots \\
G_{y}\left(\mathbf{r}_{1}, j \omega_{N_{\omega}}\right) & \cdots & G_{y}\left(\mathbf{r}_{N_{r}}, j \omega_{N_{\omega}}\right)
\end{array}\right] \in \mathbf{C}^{N_{\omega} \times N_{r}} .
$$

The dynamic response matrix

$$
\mathbf{P}^{t f}=\left[\begin{array}{ccc}
P_{1}^{-1}\left(j \omega_{1}\right) & \cdots & P_{N}^{-1}\left(j \omega_{1}\right) \\
\vdots & \ddots & \vdots \\
P_{1}^{-1}\left(j \omega_{N_{\omega}}\right) & \cdots & P_{N}^{-1}\left(j \omega_{N_{\omega}}\right)
\end{array}\right] \in \mathbf{C}^{N_{\omega} \times N}
$$

where $P_{i}^{-1}(j \omega)$ is the response of the ordered $i$ th-mode dynamics found from the system matrix $\mathbf{A}$.

$$
P_{i}^{-1}(j \omega)=\left.\frac{1}{\left[s+\left(\alpha_{i}+j \sigma_{i}\right)\right]\left[s+\left(\alpha_{i}-j \sigma_{i}\right)\right]}\right|_{s=j \omega} .
$$

The modal function matrix

$$
\Psi=\left[\begin{array}{ccc}
\Phi_{1}\left(\mathbf{r}_{1}\right) & \cdots & \Phi_{1}\left(\mathbf{r}_{N_{r}}\right) \\
\vdots & \ddots & \vdots \\
\Phi_{N}\left(\mathbf{r}_{1}\right) & \cdots & \Phi_{N}\left(\mathbf{r}_{N_{r}}\right)
\end{array}\right] \in \mathbf{R}^{N \times N_{r}} .
$$

The feed-through vector

$$
\mathbf{D}=\left[\begin{array}{lll}
D\left(\mathbf{r}_{1}\right) & \cdots & D\left(\mathbf{r}_{N_{r}}\right)
\end{array}\right] \in \mathbf{R}^{1 \times N_{r}} .
$$

We can form the following complex matrix equation:

$$
\mathbf{G}=\left[\begin{array}{ll}
\mathbf{P}^{t f} & 1_{N_{\omega} \times 1}
\end{array}\right]\left[\begin{array}{c}
\widehat{\Psi} \\
\widehat{\mathbf{D}}
\end{array}\right] \text {. }
$$

Equation (36) has a unique least squares solution if $N_{\omega} \geqslant N$, this condition is automatically satisfied if the restrictions for the subspace estimation in Section IV are met, i.e., if $N_{\omega} \geqslant q+p$, where $p$ is the model order and $q$ is the auxiliary order [31]. Since we are interested in real-valued functions, we restrict the matrices $\widehat{\Psi}$ and $\widehat{D}$ accordingly.

\section{B. Linear Reconstruction}

Here the ordering and dimension of the coordinate vector $\mathbf{r}$ becomes important. For notational simplicity, we assume $\mathbf{r}$ is single dimensional. Shannon's formula for linear reconstruction can be restated in context

$$
\begin{aligned}
\Phi_{i}(r)= & \Delta r \sum_{k=0}^{N_{x}} \Phi_{i}\left(r_{k}\right) \frac{\sin \left(\frac{\pi}{\Delta r}\left(r-r_{k}\right)\right)}{\pi\left(r-r_{k}\right)} \\
= & {\left[\Phi_{i}\left(r_{1}\right) \cdots \Phi_{i}\left(r_{N_{r}}\right)\right] } \\
& \times\left[\begin{array}{c}
\operatorname{sinc}\left(\frac{\pi}{\Delta r}\left(r-r_{1}\right)\right) \\
\vdots \\
\operatorname{sinc}\left(\frac{\pi}{\Delta r}\left(r-r_{N_{r}}\right)\right)
\end{array}\right] .
\end{aligned}
$$

Similarly for $D(r)$. For convenience, we write a relation describing all spatial functions

$$
\begin{aligned}
{\left[\begin{array}{c}
\Phi_{i}(r) \\
\vdots \\
\Phi_{N}(r) \\
D(r)
\end{array}\right] } & =\left[\begin{array}{c}
\widehat{\Psi} \\
\widehat{\mathbf{D}}
\end{array}\right]\left[\begin{array}{c}
\operatorname{sinc}\left(\frac{\pi}{\Delta r}\left(r-r_{1}\right)\right) \\
\vdots \\
\operatorname{sinc}\left(\frac{\pi}{\Delta r}\left(r-r_{N_{r}}\right)\right)
\end{array}\right] \\
& =[\widehat{\widehat{\Psi}}] \mathbf{B}_{r}(r)
\end{aligned}
$$




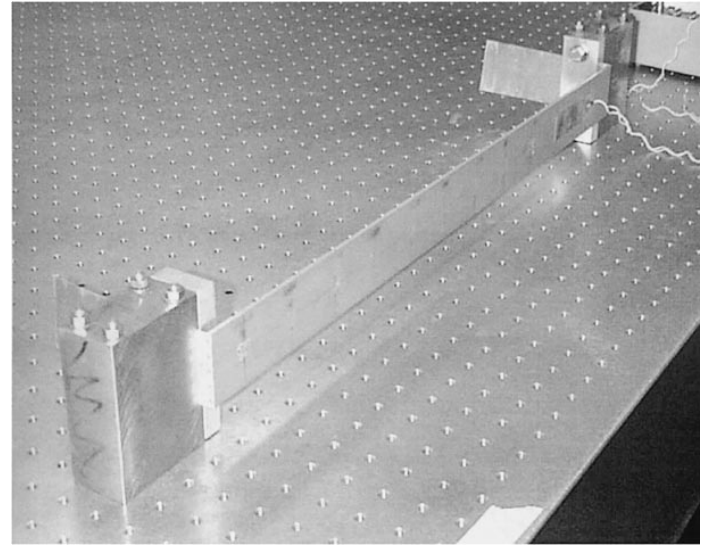

Fig. 5. Experimental beam apparatus.

where $\mathbf{B}_{r}(r)$ is the basis of reconstruction.

The spatial system can be written in state-space form

$$
\begin{aligned}
\dot{\mathbf{x}} & =\mathbf{A} \mathbf{x}+\mathbf{B} u \\
Y(r) & =\mathbf{B}_{r}(r)^{T} \widehat{\Psi}^{T} J \mathbf{x}+\widehat{\mathbf{D}} \mathbf{B}_{r}(r) u
\end{aligned}
$$

where $J=\left[\begin{array}{llll}e_{1}^{T} & e_{3}^{T} & \cdots & e_{(2 N-1)}^{T}\end{array}\right]^{T} \in \mathbf{R}^{N \times 2 N}$, and $e_{i}$ is the $i$ shifted unit impulse, e.g., $e_{3}=\left[\begin{array}{llllll}0 & 0 & 1 & 0 & \cdots & 0\end{array}\right]$. Note the equivalence of system (39) to (7), where $\mathbf{B}_{r}(r)^{T} \widehat{\Psi}^{T} J$ and $\widehat{\mathbf{D B}}_{\mathbf{r}}(r)$ represent the identified function matrix $\mathbf{C}(\mathbf{r})$ and feed-through function $\mathbf{D}(\mathbf{r})$.

\section{Spline Reconstruction}

The spline reconstructed system is similar to (39) with the exception that the function samples $\left[\begin{array}{c}\widehat{\Psi} \\ \widehat{\mathbf{D}}\end{array}\right]$ and reconstruction basis $\mathbf{B}_{r}$ are replaced by the spline coefficients and chosen spline basis.

1) Finding the Spline Coefficients $c(k)$ : Many standard procedures exist for finding the spline coefficients $c(k)$ as defined in (12). The reader is referred to [21] for an overview of such techniques.

2) Summary: After computing the spline coefficients for each mode, the spatial system can be expressed in state-space form

$$
\begin{aligned}
\dot{\mathbf{x}} & =\mathbf{A} \mathbf{x}+\mathbf{B} u \\
Y(r) & =B^{n}(r)^{T} \mathbf{C}_{s}^{T} J \mathbf{x}+\mathbf{D}_{s} B^{n}(r) u,
\end{aligned}
$$

where, $\mathbf{D}_{\mathbf{s}}=\left[\begin{array}{lll}c_{d}(1) & c_{d}(2) & c_{d}\left(N_{r}\right)\end{array}\right]$ the spline coefficients of the feed-through function $D(r), \mathbf{C}_{\mathbf{s}}$ is the matrix containing the spline coefficients for each mode, and $B^{n}(r)$ is the spline reconstruction basis

$$
\begin{aligned}
\mathbf{C}_{s}= & {\left[\begin{array}{ccc}
c_{1}(1) & \cdots & c_{1}\left(N_{r}\right) \\
\vdots & \ddots & \vdots \\
c_{N}(1) & \cdots & c_{N}\left(N_{r}\right)
\end{array}\right] } \\
B^{n}(r)= & {\left[\begin{array}{c}
\beta^{n}\left(\frac{r}{\Delta r}\right) \\
\beta^{n}\left(\frac{r}{\Delta r}-1\right) \\
\vdots \\
\beta^{n}\left(\frac{r}{\Delta r}-\left(N_{r}-1\right)\right)
\end{array}\right] . }
\end{aligned}
$$

TABLE I

BEAM PARAMETERS

\begin{tabular}{c|c}
\hline Length, $L$ & $0.6 \mathrm{~m}$ \\
\hline Width, $w_{b}$ & $0.05 \mathrm{~m}$ \\
\hline Thickness, $h_{b}$ & $0.003 \mathrm{~m}$ \\
\hline Young's Modulus, $E_{b}$ & $65 \times 10^{9} \mathrm{~N} / \mathrm{m}^{2}$ \\
\hline Density, $\rho$ & $2650 \mathrm{~kg} / \mathrm{m}^{2}$ \\
\hline
\end{tabular}

TABLE II

IDENTIFICATION PARAMETERS

\begin{tabular}{l|l}
\hline Frequency Range & $10-200(\mathrm{~Hz})$ \\
\hline Eqi-distance F Samples & 3031 \\
\hline Spatial Sampling interval & $2.5 \mathrm{~cm}$ \\
\hline Identification Samples & 13 \\
\hline Validation Samples & 13 \\
\hline Excitation & Colored Noise
\end{tabular}

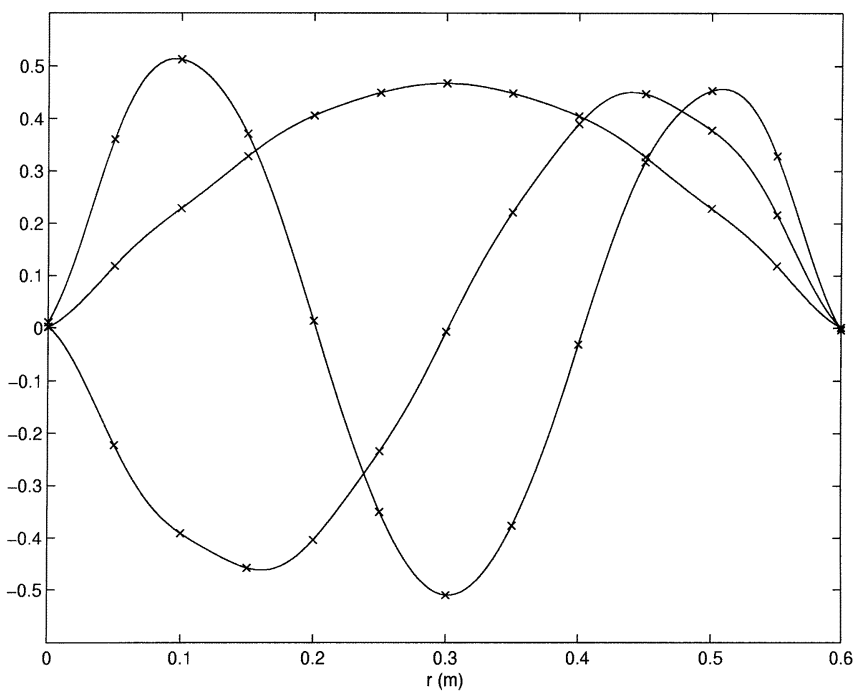

Fig. 6. Extracted mode samples $(x)$ and linear reconstruction.

\section{EXPERIMENTAL RESULTS}

The presented technique will now be applied to identify two spatially distributed systems, a simply supported beam, and asymmetric cantilever plate. Both structures are excited using bonded piezoelectric actuators. Although the simply supported beam is easily modeled using analytic methods (albeit with experimental tuning), applying such techniques to the plate is significantly more difficult. The problem is complicated by the irregular geometry of the plate boundary.

The experimental beam and plate apparatus are shown in Figs. 5 and 12.

\section{A. Beam Identification}

1) Experimental Setup: The physical parameters of interest are summarized in Table I.

Colored noise is applied to the actuator, the spatial response is measured sequentially using a Polytec scanning laser vibrometer. Details of the data set are given in Table II.

2) Spatial Functions: The extracted mode and feed-through function samples together with their spline and linear reconstructions are shown in Figs. 6-8. It can be observed that the 


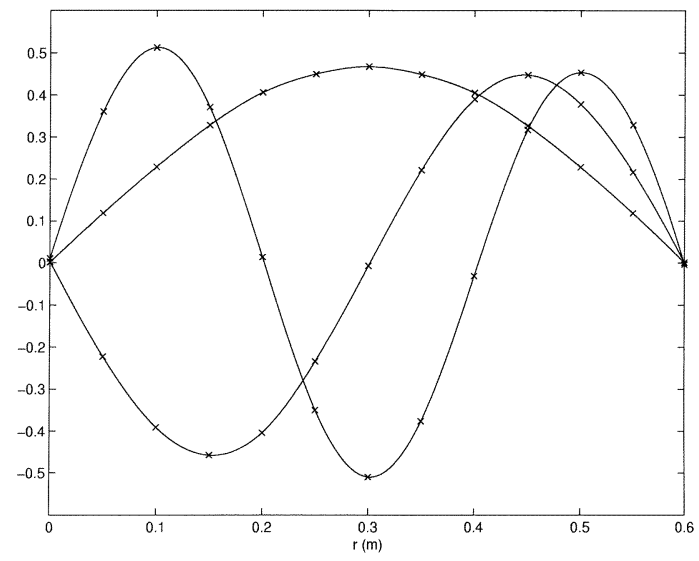

Fig. 7. Extracted mode samples $(x)$ and spline reconstruction.

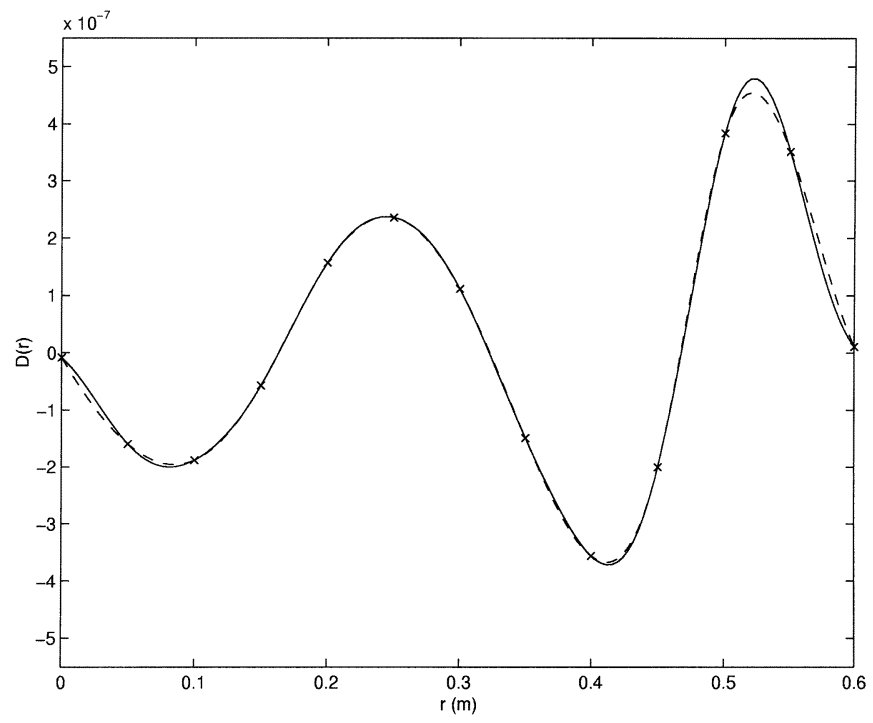

Fig. 8. Extracted feed-through function samples $(x)$, the linear reconstruction $(-)$, and spline reconstruction (- $)$.

identified feed-though function is similar to that derived analytically, shown in Fig. 3.

The Shannon reconstructed mode shapes are significantly distorted by the combination of truncation error and sample noise. It is interesting to note the effect of piezoelectric stiffness on the mode shapes of the beam shown in Fig. 7, the length of the beam bonded to the piezoelectric patch is obviously more restricted in its deflection. Such structures with localized changes in stiffness are very difficult to model in closed form using present analytic techniques.

3) Spatial Response: To evaluate model quality, we will compare the measured spatial beam response plotted in Fig. 9, to the identified model response plotted in Fig. 10. Each point-wise frequency response is measured from the applied actuator voltage (in volts) to the resulting displacement (in meters). A separate interlaced set of 13 points was used to perform the validation. The magnitude response of the error system, $G_{y}(r, j \omega)-\hat{G}_{y}(r, j \omega)$, where $\hat{G}_{y}(r, j \omega)$ denotes the model response, is plotted in Fig. 11. In the frequency domain,

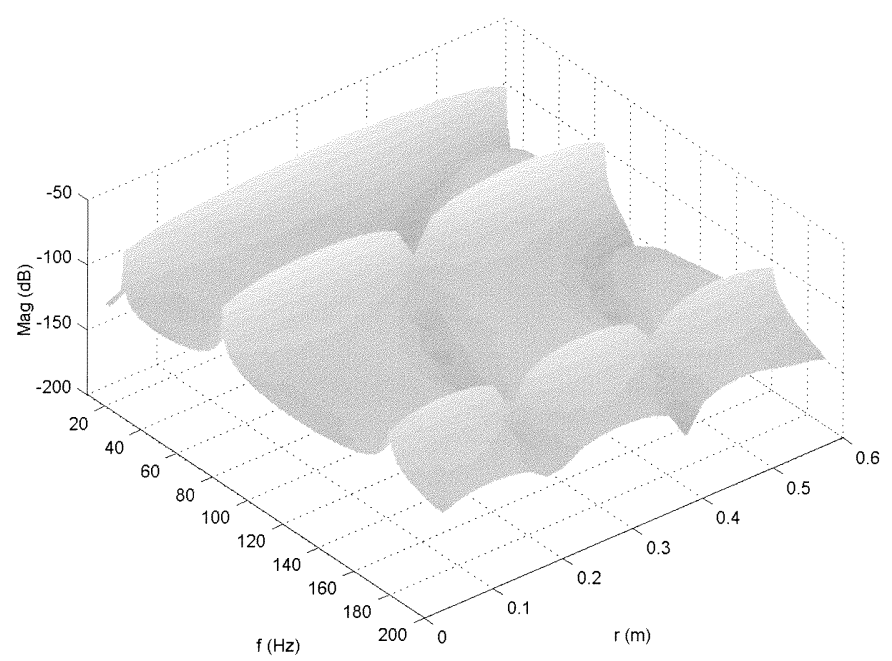

Fig. 9. Experimental beam spatial frequency response from an applied actuator voltage to the measured displacement $G_{y}(r, j \omega)$.

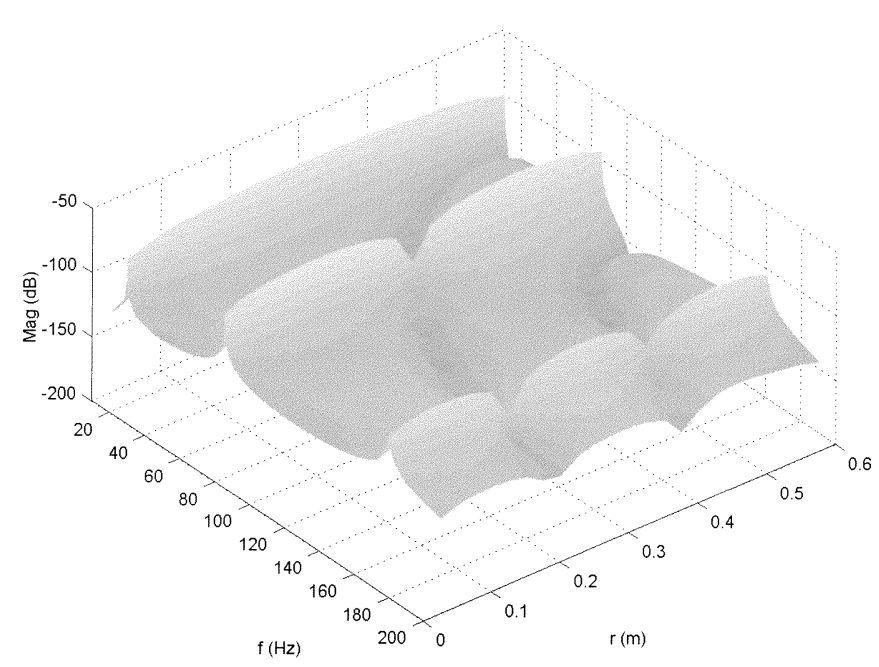

Fig. 10. Spline reconstructed model response, from an applied actuator voltage to the measured displacement $\hat{G}_{y}(r, j \omega)$.

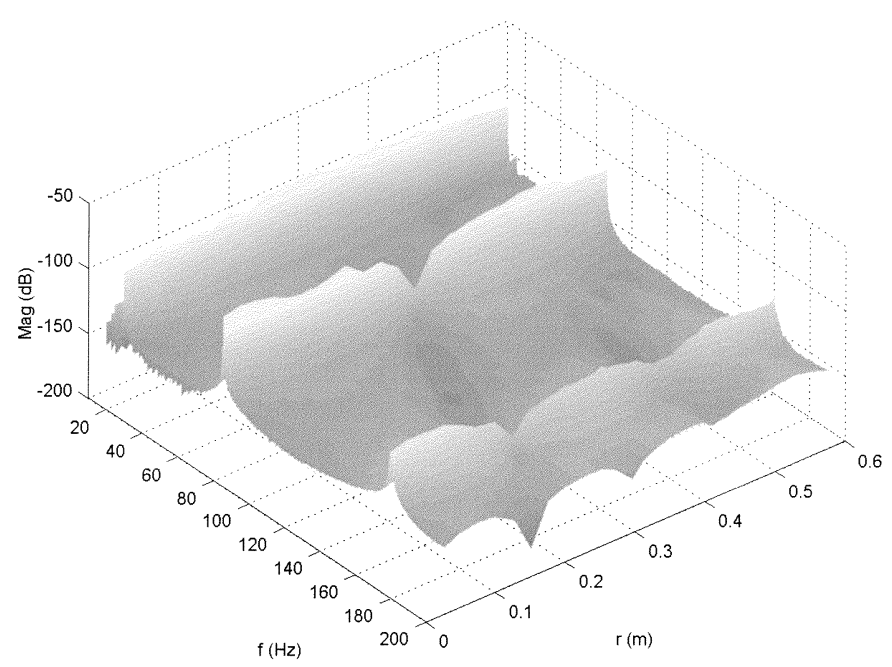

Fig. 11. Error system response response, $G_{y}(r, j \omega)-\hat{G}_{y}(r, j \omega)$.

the identified model is observed to accurately represent the physical system. 


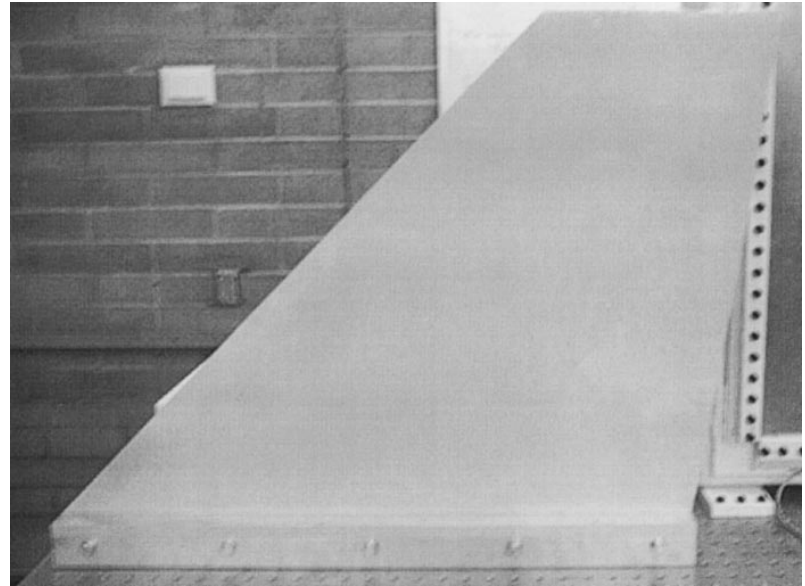

Fig. 12. Experimental plate apparatus.

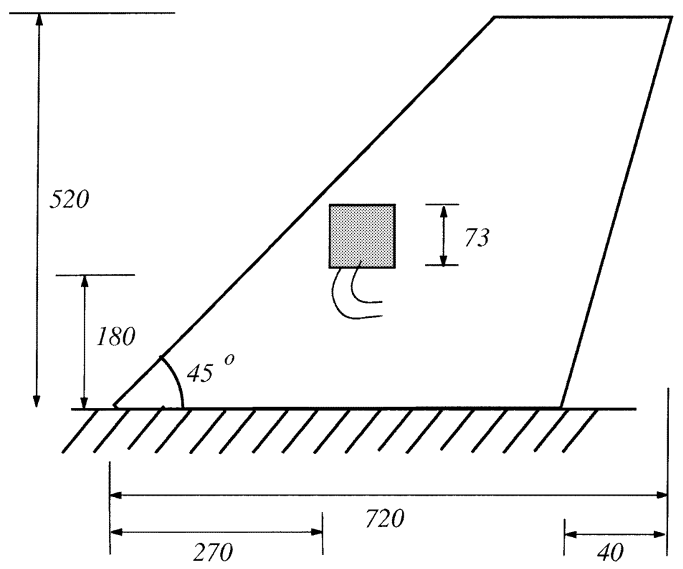

Fig. 13. Plate geometry (mm).

TABLE III

Plate System IDENTIFICATION PARAMETERS

\begin{tabular}{l|l}
\hline Frequency Range & $10-100(\mathrm{~Hz})$ \\
\hline Eqi-distance F Samples & 577 \\
\hline Number of Spatial Samples & 468 \\
\hline Spatial Sampling interval & $2.63 \mathrm{~cm}$ \\
\hline Excitation & Colored Chirp \\
\hline
\end{tabular}

\section{B. Plate Identification}

1) Experimental Setup: The experimental plate is constructed from aluminum of $4 \mathrm{~mm}$ thickness. Fig. 12 shows the experimental plate, clamped vertically by its bottom edge to an optical table. Geometry and dimensions are shown in Fig. 13. System identification parameters are given in Table III.

2) Spatial Functions: An estimate for the system matrix $\mathbf{A}$ is first obtained using a scattered subset of the spatial frequency samples. The location of subset points is shown in Fig. 14. Equation (36) is solved to identify the mode shapes and feed-through function. The resulting mode shapes and feed-through function are plotted in Figs. 15 and 16.

3) Spatial Response: Due to the difficulties in visualizing a four-dimensional quantity, we evaluate model quality by taking a planar section of the spatial frequency response. An elevation of the section is shown in Fig. 14. The measured, identified model, and error system $G_{y}(r, j \omega)-\hat{G}_{y}(r, j \omega)$ fre-

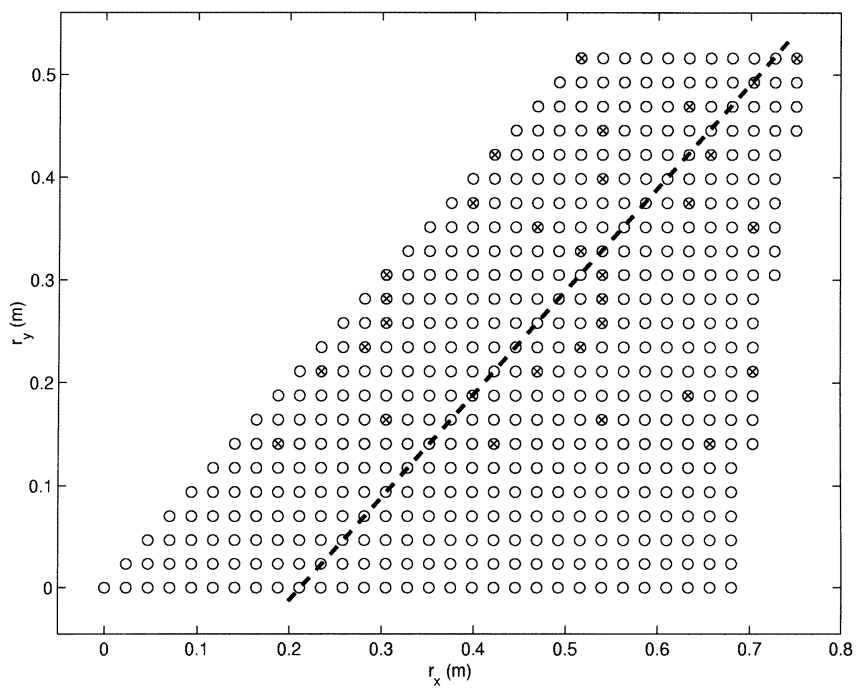

Fig. 14. Distribution of the spatial samples. An " $\times$ " represents the location of a sample used to identify the system matrix A. The dashed line represents the side elevation of a spatial frequency response cross section used to analyze model quality.

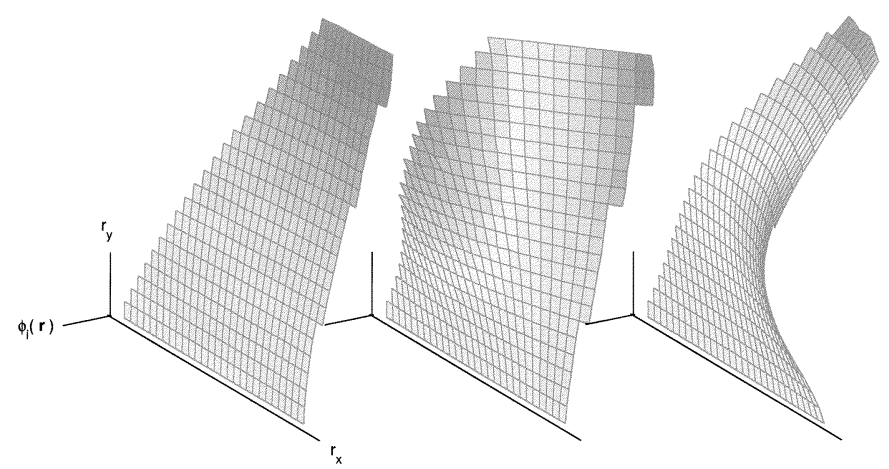

Fig. 15. Normalized first, second, and third modes of the cantilever fin.

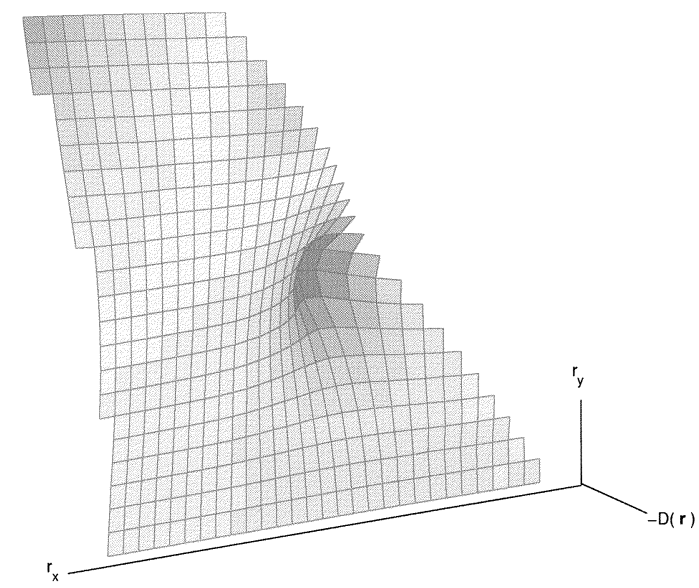

Fig. 16. Identified feed-through function $D(\mathbf{r})$.

quency responses are shown in Figs. 17-19, respectively. Each point-wise frequency response is measured from the applied actuator voltage to the resulting displacement (in meters). As shown by the magnitude of the error system response, in the frequency domain, a good correlation between the experimental data and model response is observed. 


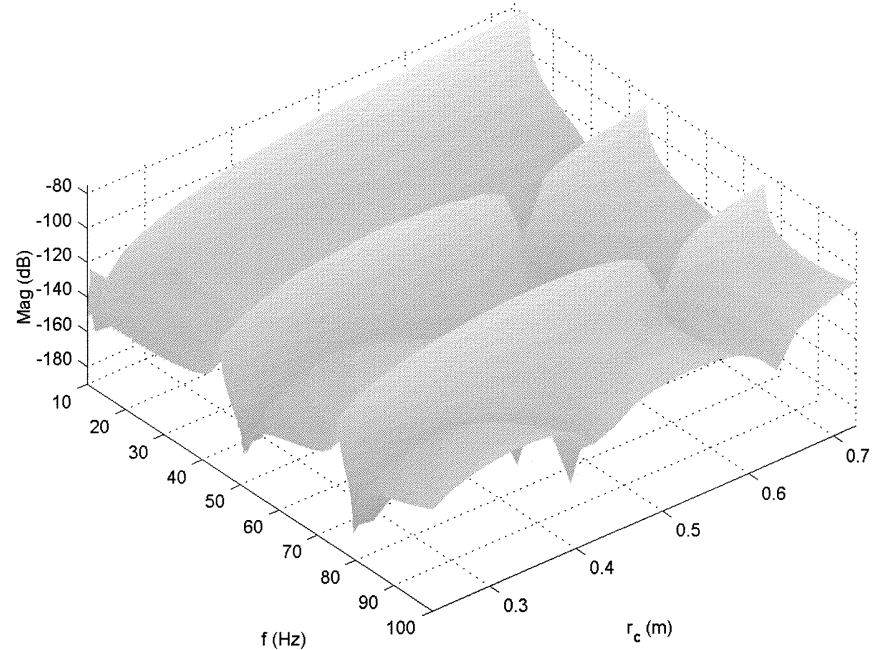

Fig. 17. Cross section of the cantilever plate measured spatial frequency response. The response is measured from the applied actuator voltage to the resulting displacement.

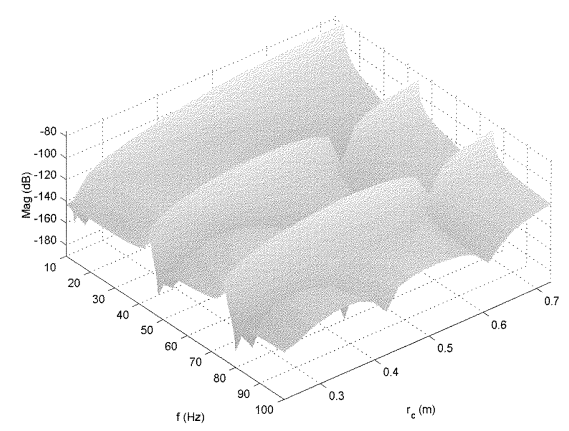

Fig. 18. Cross section of the spline reconstructed model response.

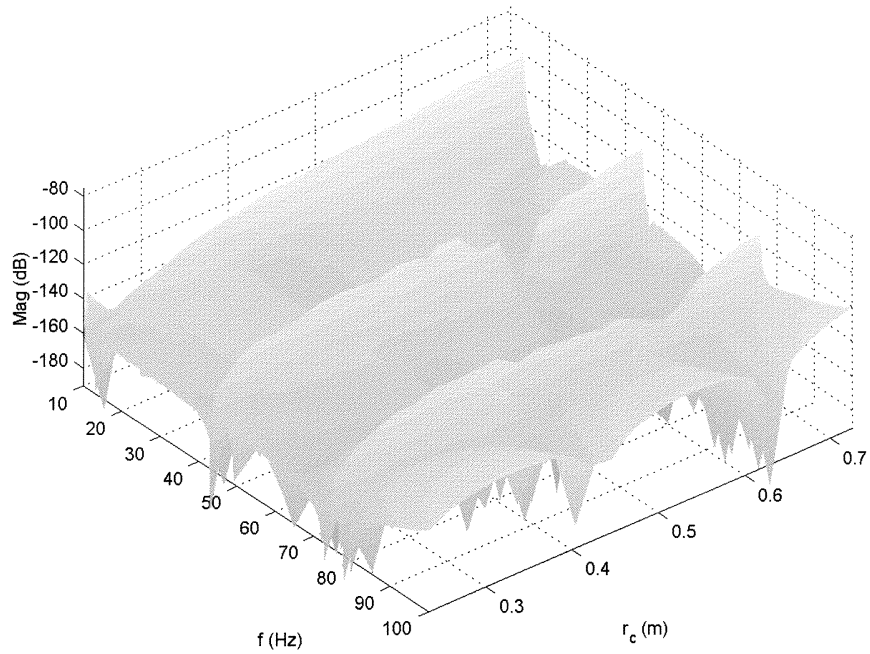

Fig. 19. Cross-section of the plate error system frequency response, $G_{y}(r, j \omega)-\hat{G}_{y}(r, j \omega)$.

\section{CONCLUSION}

A technique has been presented for identifying a class of distributed parameter systems from a set of spatially distributed frequency responses. The systems are modeled as a finite sum of second-order transfer functions with spatially variant numerators and a feed-through term.
In an attempt to evenly distribute model error, the identification is cast as a single-input multi-output identification problem. An estimate for the system dynamics is sought using a frequency domain subspace algorithm. Samples of the mode shapes and feed-through function are then identified and used to reconstruct the continuous functions. If the spatial Fourier transform is known, the error due to under sampling can be quantified.

Experimental identification of a simply supported beam and cantilever plate has shown an adequate correlation in the frequency domain between the measured system and identified model. In both cases, the majority of discrepancy is due to small errors in the resonance frequencies. Current work involves the development of an efficient optimization algorithm to minimize such errors.

Other topics of current research include: the automatic identification of nondistributed dynamics $H(s)$, experimental identification incorporating piezoelectric sensor voltages, time domain identification techniques, and stochastic analysis.

\section{ACKNOWLEDGMENT}

The authors would like to acknowledge S. Behrens and Dr. B. Ninness for their contributions in respect to construction of the experimental apparatus and system identification software.

\section{REFERENCES}

[1] D. Halim and S. 0. Reza Moheimani, "Experimental implementation of spatial $h$-infinity control on a piezoelectric laminate beam," IEEE/ASME Trans. Mechatron., vol. 7, pp. 346-356, Sept. 2002.

[2] S. Skogestad and I. Postlethwaite, Multivariable Feedback Control. New York: Wiley, 1996.

[3] K. Zhou, J. C. Doyle, and K. Clover, Robust and Optimal Control. Upper Saddle River, NJ: Prentice-Hall, 1996.

[4] S. 0. Reza Moheimani, H. R. Pota, and I. R. Petersen, "Broadband disturbance attenuation over an entire beam," J. Sound Vibration, vol. 227, no. 4, pp. 807-832, 1999.

[5] D. Halim and S. 0. Reza Moheimani, "Spatial resonant control of flexible structures-Application to a piezoelectric laminate beam," IEEE Trans. Contr. Syst. Technol., vol. 9, pp. 37-53, Jan. 2001.

[6] — - "Spatial $H_{2}$ control of a piezoelectric laminate beam: Experimental implementation," IEEE Trans. Contr. Syst. Technol., vol. 10, pp. 533-546, July 2002.

[7] L. Meirovitch, Elements of Vibration Analysis, 2 ed. New York: McGraw-Hill, 1996.

[8] A. R. Fraser and R. W. Daniel, Pertubation Techniques for Flexible Manipulators. Boston, MA: Kluwer, 1991.

[9] J. Heng, J. C. Akers, R. Venugopal, M. Lee, A. C. Sparks, P. D. Washabaugh, and D. Bernstien, "Modeling, identification, and feedback control of noise in an acoustic duct," IEEE Trans. Contr. Syst. Technol., vol. 4, pp. 283-291, May 1996.

[10] R. D. Cook, Finite Element Modeling for Stress Analysis. New York: Wiley, 1995.

[11] Y. Lim, V. V. Varadan, and V. K. Varadan, "Closed-loop finite element modeling of active/passive damping in structural vibration control," Proc. SPIE Smart Materials Structures, Mathematics, Control Smart Structures, vol. 3039, Mar. 1997.

[12] D. J. Ewins, "Modal testing as an aid to vibration analysis," in Proc. Conf. Mechanical Engineering, May 1990.

[13] N. M. M. Maia and J. M. Montalvao de Silva, Eds., Theoretical and Experimental Modal Analysis. Hertfordshire, U.K.: Research Studies Press, 1997.

[14] S. 0. Reza Mohiemani, "Minimizing the effect of out-of-bandwidth dynaimes in the models of reverberant systems that arise in modal analysis: Implications on spatial $H_{\infty}$ control," Automatica, vol. 36, pp. 1023-1031, 2000.

[15] A. J. Jerri, "The shannon sampling theorem-Its various extensions and applications: A tutorial review," Proc. IEEE, vol. 65, pp. 1565-1596, Nov. 1977. 
[16] M. Pawlak and E. Rafajlowicz, "On restoring band-limited signals," IEEE Trans. Inform. Theory, vol. 40, pp. 1490-1503, Sept. 1994.

[17] M. Pawlak and U. Stadtmüller, "Recovering band-limited signals under noise," IEEE Trans. Inform. Theory, vol. 42, pp. 1425-1438, Sept. 1996.

[18] A. Krzyzak and E. R. M. Pawlak, "Moving average restoration of bandlimited signals from noisy observations," IEEE Trans. Signal Processing, vol. 45, pp. 2967-2976, Dec. 1997.

[19] J. B. Thomas and B. Liu, "Error problems in sampling representation," IEEE Int. Conv. Rec. (USA), vol. 12, no. 5, pp. 269-277, 1964.

[20] P. Lancaster and K. Salkauskas, Curve and Surface Fitting. New York: Academic, 1986.

[21] M. Unser, "Splines, a perfect fit for signal and image processing," IEEE Signal Processing Mag., vol. 16, pp. 22-38, Nov. 1999.

[22] M. Unser, A. Aldroubi, and M. Eden, "Polynomial spline signal approximations: Filter design and asymptotic equivalence with shannon's sampling theorem," IEEE Trans. Inform. Theory, vol. 38, pp. 95-103, Jan. 1992.

[23] R. Hummel, "Sampling for spline reconstruction," SIAM J. Appl. Math., vol. 43, pp. 278-288, Apr. 1983.

[24] T. Blu and M. Unser, "Quantitative fourier analysis of approximation techniques: Part 1-Interpolators and projectors," IEEE Trans. Signal Processing, vol. 47, pp. 2783-2795, Oct. 1999.

[25] I. J. Schoenberg, Cardinal Spline Interpolation. Philadelphia, PA: Soc. Ind. Appl. Math., 1973.

[26] A. D. Poularikas, Handbook of Formulas and Tables for Signal Processing. Boca Raton, FL: CRC, 1999.

[27] R. L. Fante, Signal Analysis and Estimation. An Introduction, New York: Wiley, 1988.

[28] S. 0. Reza Moheimani, "Experimental verification of the corrected transfer function of a piezoelectric laminate beam," IEEE Trans. Contr. Syst. Technol., vol. 8, pp. 660-666, July 2000.

[29] M. Viberg, "Subspace-based methods for the identification of linear time invariant systems," Automatica, vol. 31, no. 12, pp. 1835-1851, 1995.

[30] K. Liu, R. N. Jacques, and D. W. Miller, "Frequency domain structural system identification by observability range space extraction," in Proc. American Control Conf., vol. 1, Baltimore, MD, June 1994, pp. 107-111.

[31] T. McKelvy, H. Akcay, and L. Ljung, "Subspace based multivariable system identification from frequency response data," IEEE Trans. Automat. Contr., vol. 41, pp. 960-978, July 1996.

[32] T. McKelvey, A. J. Fleming, and S. 0. Reza Moheimani, "Subspace based system identification for an acoustic enclosure," ASME J. Vibr. Acoust., vol. 124, pp. 414-419, July 2002.

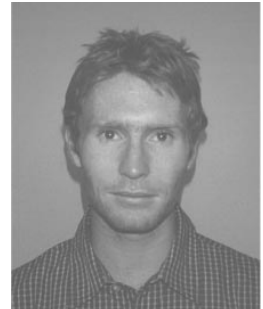

Andrew J. Fleming was born in Dingwall, U.K., in 1977. He received the B.E. (Elec.) degree from The University of Newcastle, Callaghan, Australia, with in 2000 and is currently persuing the Ph.D. degree in the School of Electrical Engineering and Computer Science at the same university.

$\mathrm{He}$ is a member of the Centre for Integrated Dynamics and Control, an Australian Government Special Research Centre. He is also a Member of the Laboratory for Dynamics and Control of Smart Structures, which is funded by the Australia Research Council. His research involves passive, semiactive, and active-shunt vibration control of piezoelectric and electromagnetically actuated structures.

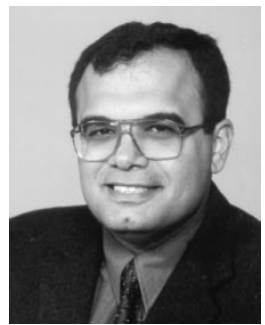

S. O. Reza Moheimani (S'93-M'97-SM'00) was born in Shiraz, Iran, in 1967. He received the B.Sc. degree from Shiraz University in 1990 and the M.Eng.Sc. and Ph.D. degrees from the University of New South Wales, Australia, in 1993 and 1996, respectively, all in electrical and electronics engineering.

In 1996, he was a Postdoctoral Research Fellow at the School of Electrical and Electronics Engineering, Australian Defence Force Academy, Canberra, Australia. In 1997, he joined the University of Newcastle, Callaghan, Australia where he is currently a Senior Lecturer in the School of Electrical Engineering and Computer Science. He is a coauthor of the research monograph Spatial Control of Vibration: Theory and Experiments (Singapore: World Scientific, 2003) and the Editor of volume Perspectives in Robust Control (Berlin, Germany: Springer-Verlag, 2001). He has authored/coauthored over 100 technical papers. He is a Member of IFAC Technical Committee on Mechatronic Systems. His research interests include smart structures, mechatronics, control theory, and signal processing. He is an Associate Editor for Control Engineering Practice, and International Journal of Control, Automation, and Systems.

Dr. Reza Moheimani has served on the editorial boards of several international conferences, and is the Chairman of International Program Committee for the IFAC Conference on Mechatronic Systems, to be held in Sydney, Australia, in September 2004. 